\title{
GLOBAL BIFURCATION OF SOLITARY WAVES TO THE BOUSSINESQ abcd SYSTEM
}

\author{
ROBIN MING CHEN AND JIE JIN
}

\begin{abstract}
The Boussinesq abcd system arises in the modeling of long wave small amplitude water waves in a channel, where the four parameters $(a, b, c, d)$ satisfy one constraint. In this paper we focus on the solitary wave solutions to such a system. In particular we work in two parameter regimes where the system does not admit a Hamiltonian structure (corresponding to $b \neq d$ ). We prove via analytic global bifurcation techniques the existence of solitary waves in such parameter regimes. Some qualitative properties of the solutions are also derived, from which sharp results can be obtained for the global solution curves.

Specifically, we first construct solutions bifurcating from the stationary waves, and obtain a global continuous curve of solutions that exhibits a loss of ellipticity in the limit. The second family of solutions bifurcate from the classical Boussinesq supercritical waves. We show that the curve associated to the second class either undergoes a loss of ellipticity in the limit or becomes arbitrarily close to having a stagnation point.
\end{abstract}

\section{CONTENTS}

1. Introduction

2. Bifurcation from stationary waves with $a=c<0$

3. Bifurcation from classical Boussinesq supercritical waves 17

Acknowledgements $\quad 22$

Appendix A. Proofs from Section $2.1 \quad 23$

References $\quad 24$

\section{INTRODUCTION}

The phenomenon of solitary wave was first observed by John Scott Russel [32] almost two centuries ago, which is later used to characterize wave that does not disperse and retains its original identity as time evolves. Exact existence theory for solitary water waves, however, first appeared more than a century later in the work of Lavrentiev [29], Friedrichs-Hyers [24], and Ter-Krikorov [37] for small-ampliltude irrotational waves. Construction for large-amplitude irrotational waves was achieved by Amick-Toland [4, 5] and Benjemin-Bona-Bose [7].

Russel's experiment also motivated the studies on the mathematical modeling of water waves. The first works can be dated back to Boussinesq [12, Rayleigh [31, Korteweg and de Vries [28, where simpler sets of equations were derived as asymptotic models from the free surface Euler equations in some specific physical regimes. To 
be more precise, let $h$ and $\lambda$ denote respectively the mean elevation of the water over the bottom and the typical wavelength, and let $a$ be a typical wave amplitude. The parameter regime considered in the above works corresponds to

$$
\varepsilon=\frac{a}{h} \ll 1, \quad \delta=\frac{h}{\lambda} \ll 1, \quad \varepsilon=O\left(\delta^{2}\right),
$$

which is called the small amplitude, shallow water regime. Physically, $\varepsilon$ measures the strength of nonlinearity while $\mu$ characterizes the effect of dispersion. Thus solitary waves can be viewed as generated from a perfect balance between nonlinear and dispersive effects. The reduced systems within the above scaling regime couple the free surface elevation $\eta$ to the horizontal component of the velocity $u$, and include the celebrated KdV equation and the Boussinesq equation [12, 22, 26, 28, 39].

In this paper we will consider solitary wave solutions to an asymptotic water wave model derived by Bona-Chen-Saut [9] (generalized to include the surface tension in [23] and in higher dimensions Bona-Colin-Lannes [10]) as an extended system of the classical Boussinesq equation. Specifically, it is a three-parameter family of Boussinesq systems for one dimensional surfaces that takes the following form

$$
\left\{\begin{array}{l}
\eta_{t}+u_{x}+(u \eta)_{x}+a u_{x x x}-b \eta_{x x t}=0 \\
u_{t}+\eta_{x}+\frac{1}{2}\left(u^{2}\right)_{x}+c \eta_{x x x}-d u_{x x t}=0
\end{array}\right.
$$

all of which are formally equivalent models of solutions of the Euler equations. In the above system $\eta$ is proportional to the deviation of the free surface from its rest position, $u$ is proportional to the horizontal velocity taken at the scaled height $0 \leq \theta \leq 1(\theta=1$ at the free surface and $\theta=0$ at the bottom). The parameters have the following explicit form

$a=\left(\frac{\theta^{2}}{2}-\frac{1}{6}\right) \nu, \quad b=\left(\frac{\theta^{2}}{2}-\frac{1}{6}\right)(1-\nu), \quad c=\frac{\left(1-\theta^{2}\right)}{2} \mu-\tau, \quad d=\frac{\left(1-\theta^{2}\right)}{2}(1-\mu)$

with $\nu$ and $\mu$ arbitrary real numbers, and $\tau \geq 0$ is the normalized surface tension. These three degrees of freedom arise from the height at which the horizontal velocity is taken and from a double use of the BBM trick [8]. The hydrodynamic relevance of the model was justified in [10, 14, 34.

A solitary wave solution to system (1.1) is of the type

$$
\eta(x, t)=\eta(\xi)=\eta(x-\lambda t) \in H^{1}(\mathbb{R}), \quad u(x, t)=u(\xi)=u(x-\lambda t) \in H^{1}(\mathbb{R}),
$$

where $\lambda$ denotes the traveling speed and $\xi=x-\lambda t$ is the moving coordinate with speed $\lambda \in \mathbb{R}$. We are thus looking in the class of "localized" solutions to the system

$$
\left\{\begin{array}{l}
c \eta^{\prime \prime}+\eta-\lambda u+d \lambda u^{\prime \prime}+\frac{1}{2} u^{2}=0 \\
a u^{\prime \prime}+u-\lambda \eta+b \lambda \eta^{\prime \prime}+\eta u=0
\end{array}\right.
$$

where / denotes the derivative with respect to $\xi$. The regularity of the solutions indicates the asymptotic behavior

$$
\lim _{|x| \rightarrow \infty}(\eta, u)=(0,0)
$$

Note that when $b=d$, the system possesses a Hamiltonian structure with Hamiltonian

$$
\mathcal{H}(\eta, u)=\frac{1}{2} \int\left[-c \eta_{x}^{2}-a u_{x}^{2}+\eta^{2}+(1+\eta) u^{2}\right] d x
$$


The solitary waves correspond to the critical points of the action functional $S_{\lambda}=$ $\mathcal{H}-\lambda \mathcal{I}$, where

$$
\mathcal{I}(\eta, v)=\int\left(\eta u+b \eta_{x} u_{x}\right) d x
$$

is called the impulse functional, and the Lagrange multiplier $\lambda$ gives the speed of the wave.

From (1.5) we see that the Hamiltonian $\mathcal{H}(\eta, u)$ is coercive in $H^{1}$ provided that $a, c<0$. In this parameter regime, the existence of solitary waves can be inferred from the existence of minimizers to a constraint minimization problem [16] under the assumptions that the surface tension is large $(\tau>1 / 3)$ and $\|\eta\|_{H^{2}}$ is small. Later in [17] another variational formulation was adapted in the same parameter regime to establish the existence of solitary waves for any $\tau \geq 0$, but with a smallness restriction on the traveling speed $\lambda$. Using a Nehari manifold technique, the existence of ground state solutions (nontrivial solitary waves carrying minimum action energy $S_{\lambda}$ ) was established in [6]. In the case of large surface tension $\tau>1 / 3$, these ground states are shown to be depression waves which are symmetric and increasing from their unique troughs, consistent with the results in the context of two-dimensional full gravity-capillary water waves [1, 27, 33].

All the above analytical results are crucially based upon the Hamiltonian structure of the system, i.e., $b=d$. Our main goal is to extend the existence result to the cases when the parameters fall out of this regime. In particular, we will focus on pure gravity waves, corresponding to $\tau=0$, and allow either (i) $b \neq d$, so that the Hamiltonian structure is no longer available; (ii) $a, c>0$, so that the quadratic part of the Hamiltonian (1.5) is not positive definite; or (iii) the wave speed $\lambda$ is large $|\lambda|>1$, so that the action functional fails to be bounded from below. In all cases, the standard variational method seems hard to apply.

The main tool we are using is the bifurcation theory. For this to work we need to first choose a good parameter $s \in \mathbb{R}$ with which the problem (1.3) can be formulated as an abstract one-parameter problem

$$
\mathscr{F}(U, s)=0
$$

where $U:=(u, \eta)$. The perturbative construction of solutions relies on a good understanding of the linearized operator $\mathscr{F}_{U}$ at some special solution $\left(U_{0}, s_{0}\right)$. It turns out that the translation invariance of the problem naturally generates a nontrivial kernel of the linearized operator $\mathscr{F}_{U}$ at any solution. With some appropriate choices of the "base point solution" $\left(U_{0}, s_{0}\right)$, standard ODE techniques can be applied to ensure that the kernel is exactly one dimensional and hence can be removed by suitable choice of the function spaces, allowing us to invoke the Implicit Function Theorem to obtain a local curve of solutions.

As is common for the solitary wave problem, continuing the local curve globally by standard global bifurcation techniques faces a serious obstruction due to the unboundedness of the domain. One classical approach is to approximate the solitary waves by periodic ones as the period tends to infinity. Such a method is used by Toland [38] to treat (1.3) with $(a, b, c, d)=\left(0, \frac{1}{3},-\frac{1}{3}, \frac{1}{3}\right)$. He first obtains a global bifurcation theory for the periodic problems, and then proves a uniform estimate. Together with an application of the Whyburn lemma, this leads to the convergence of the global sets 
of periodic solutions to a global connected set of solitary wave solutions as the period goes to infinity.

We will adapt a recently developed analytic global implicit function theorem in [20] for the global theory, cf. Theorem 2.5. As is pointed out in [20, the global curve may not be locally pre-compact, nor can one assume a priori that Fredholmness persists. Thus the loss of compactness emerges as an alternative. The ODE nature of the problem easily rules out the failure of Fredholmness. Therefore the theory will become useful in practice if we can rule out the loss of properness or classify how it manifests.

More specifically, we will consider global branches of solutions emanating from two base point solutions: the first one being the stationary solution (corresponding to $\lambda=0)$, and the second being the supercritical $(\lambda>1)$ waves to the classical Boussinesq system (corresponding to $\left.(a, b, c, d)=\left(0,0,0, \frac{1}{3}\right)\right)$. We will also assign different parameters when studying these two types of waves. When bifurcating from the stationary waves, we use the wave speed $\lambda$ as the bifurcation parameter while fixing the abcd system as in (2.5), and obtain a continuous curve of solutions all the way into the regime where solutions are traveling with an $O(1)$ speed. For the other case we will fix an arbitrary supercritical speed $\lambda>1$ and design a family of abcd systems (as in (3.3)) that can accommodate solitary waves with such a speed $\lambda$. In both cases we prove a collection of qualitative properties of the solutions that are crucial for the final global result. In particular, using maximum principle arguments and the symmetry result for weakly coupled cooperative elliptic systems [13] we are able to obtain local uniqueness, local monotonicity, and nodal pattern of the solutions. The fact that we are always considering a system makes the maximum arguments more delicate, and possibly more restrictive; see Section $2.2,2.3$ and Section 3.13 .2 .

Regarding the ruling-out/realization of the loss of compactness alternative in the global theory, as was studied in [19, 20, the established monotonicity property is strong enough to assert a "compactness or front" result stating that this possibility must manifest as a broadening phenomenon, leading to a monotone front type of solution at the end of the bifurcation curve. When the underlying system possesses a Hamiltonian structure, a so-called conjugate flow analysis can be carried out utilizing the conserved quantities to rule out the broadening alternative [2, 19, 20, 21, 25, 36]. Moreover, for some particular problems such a Hamiltonian structure may also allow one to obtain uniform bounds on solutions that can account for the realization of broadening [25]. In the cases we consider, however, the system is not Hamiltonian, and we do not have any obvious conserved quantities that can be of much use to control the solutions. Taking advantage of the monotonicity and together with delicate algebra we are able to prove the nonexistence of monotone front solutions, cf. Lemma 2.7 and Lemma 3.2. Using this idea we can also prevent the blowup of solutions $(u, \eta)$ in the case of bifurcation from stationary waves, which leads to a sharp result ensuring the loss of ellipticity as the only remaining alternative cf. Theorem 2.6. For the other case of solutions bifurcating from the classical Boussinesq waves, we are able to winnow the alternatives down to the possibilities of either the loss of ellipticity or that the curve continues up to the appearance of an "extreme wave" that has a stagnation point, cf. Theorem 3.4 . 


\section{Bifurcation from stationary Waves With $a=c<0$}

We start by constructing solutions near the stationary waves corresponding to $\lambda=0$. To ensure ellipticity we will impose the sign condition $a, c<0$.

2.1. Stationary solutions. Note that in the case when $\lambda=0$ the terms in system (1.3) containing $b$ and $d$ disappear and becomes

$$
\left\{\begin{array}{l}
-c \eta^{\prime \prime}=\eta+\frac{u^{2}}{2} \\
-a u^{\prime \prime}=u(1+\eta) .
\end{array}\right.
$$

By elliptic regularity we know that any solution of (2.1) is smooth and $\lim _{|x| \rightarrow \infty}\left(\eta^{\prime}, u^{\prime}\right)=$ $(0,0)$. Hence solitary wave solutions satisfy the 'first integral' property

$$
-a\left(u^{\prime}\right)^{2}-c\left(\eta^{\prime}\right)^{2}=u^{2}(1+\eta)+\eta^{2}
$$

The existence theory for (2.1) has been has been studied systematically in [18]. Here we collect some resulta that will be important for the later bifurcation argument. For the reader's convenience we provide their proofs in Appendix $\mathrm{A}$.

Lemma 2.1. Any solitary wave solution of (2.1) satisfies

$$
\eta(x)<0 \quad \text { on } \mathbb{R} \text {. }
$$

Proposition 2.1 (Existence and uniqueness of stationary waves [18]). When $a=c=$ $-\beta^{2}<0$ we have

(i) there is a solitary wave solution such that $u_{0}^{-}(x)<0$ on $\mathbb{R}$. Up to translation,

$$
u_{0}^{-}(x)=-\frac{3 \sqrt{2}}{2} \operatorname{sech}^{2}\left(\frac{x}{2 \beta}\right), \quad \text { and } \quad \eta_{0}(x)=-\frac{3}{2} \operatorname{sech}^{2}\left(\frac{x}{2 \beta}\right) .
$$

This solution is unique among the class of functions $(u, \eta)$ where $u<\sqrt{2}$;

(ii) there is a solitary wave solution such that $u_{0}^{+}(x)>0$ on $\mathbb{R}$. Up to translation,

$$
u_{0}^{+}(x)=\frac{3 \sqrt{2}}{2} \operatorname{sech}^{2}\left(\frac{x}{2 \beta}\right), \quad \text { and } \quad \eta_{0}(x)=-\frac{3}{2} \operatorname{sech}^{2}\left(\frac{x}{2 \beta}\right) .
$$

This solution is unique among the class of functions $(u, \eta)$ where $u>-\sqrt{2}$.

Note that from elliptic regularity we easily see that $\left(u_{0}^{ \pm}, \eta_{0}\right) \in H^{\infty}(\mathbb{R}) \times H^{\infty}(\mathbb{R})$.

2.2. Local theory. Now we will construct a local curve of solutions nearby the stationary solution $\left(u_{0}, \eta_{0}\right)$. The parameters we are taking satisfy

$$
a=c=-d=-\beta^{2}<0, \quad b=\frac{1}{3}+\beta^{2} .
$$

Obviously we see that $b \neq d$, and hence we are outside the Hamiltonian regime when the surface tension is small. For simplicity we will take $\tau=0$ in the following discussion. To fit our argument in the framework of [20], we will consider the problem in Hölder spaces.

Denote by $C_{0}(\mathbb{R})$ the set of continuous functions vanishing at infinity and

$$
C_{\mathrm{b}}^{2+\alpha}(\mathbb{R})=\left\{f \in C^{2}(\mathbb{R}):\|f\|_{C^{2+\alpha}}<+\infty\right\} .
$$


Define for $\alpha \in(0,1)$ the following Hölder space

$$
\begin{aligned}
& \mathscr{X}:=\left(C_{\mathrm{b}, \mathrm{e}}^{2+\alpha}(\mathbb{R}) \cap C_{0}(\mathbb{R})\right) \times\left(C_{\mathrm{b}, \mathrm{e}}^{2+\alpha}(\mathbb{R}) \cap C_{0}(\mathbb{R})\right), \\
& \mathscr{Y}:=\left(C_{\mathrm{b}, \mathrm{e}}^{\alpha}(\mathbb{R}) \cap C_{0}(\mathbb{R})\right) \times\left(C_{\mathrm{b}, \mathrm{e}}^{\alpha}(\mathbb{R}) \cap C_{0}(\mathbb{R})\right),
\end{aligned}
$$

where the subscript 'e' denotes the restriction to even functions, and The use of $C_{0}(\mathbb{R})$ is to realize the asymptotic condition (1.4).

Writing $U=(u, \eta)$, the system for solitary waves takes the following form

$$
\mathscr{F}(U, \lambda):=\left(\begin{array}{c}
\mathcal{L}\left(u-\lambda\left(1+\frac{1}{3 \beta^{2}}\right) \eta\right)+\left(\frac{\lambda}{3 \beta^{2}}+u\right) \eta, \\
\mathcal{L}(\eta-\lambda u)+\frac{1}{2} u^{2}
\end{array}\right)=0
$$

where

$$
\mathscr{F}: \mathscr{X} \rightarrow \mathscr{Y},
$$

and $\mathcal{L}:=1-\beta^{2} \partial_{x}^{2}$ is an invertible operator from $C_{\mathrm{b}, \mathrm{e}}^{2+\alpha}(\mathbb{R}) \cap C_{0}(\mathbb{R}) \rightarrow C_{\mathrm{b}, \mathrm{e}}^{\alpha}(\mathbb{R}) \cap C_{0}(\mathbb{R})$.

The discussion in Section 2.1 indicates that $\mathscr{F}\left(U_{0}^{ \pm}, 0\right)=0$ where $U_{0}^{ \pm}:=\left(u_{0}^{ \pm}, \eta_{0}\right)$. The linearized operator at the solution $\left(U_{0}^{ \pm}, 0\right)$ is

$$
\mathscr{F}_{U}\left(U_{0}^{ \pm}, 0\right)[V]=\mathcal{L} V+\left(\begin{array}{cc}
\eta_{0} & u_{0}^{ \pm} \\
u_{0}^{ \pm} & 0
\end{array}\right) V,
$$

where $V:=(v, \zeta) \in \mathscr{X}$. The following lemma states that the kernel of $\mathscr{F}_{U}\left(U_{0}^{ \pm}, 0\right)$ is only generated by the translation symmetry.

Lemma 2.2. For any given $\beta>0, \mathscr{F}_{U}\left(U_{0}^{ \pm}, 0\right): \mathscr{X} \rightarrow \mathscr{Y}$ is injective.

Proof. Let $V=(v, \zeta) \in \operatorname{ker} \mathscr{F}_{U}\left(U_{0}^{ \pm}, 0\right)$. Then $V$ satisfies

$$
\mathcal{L} V+\left(\begin{array}{cc}
\eta_{0} & u_{0}^{ \pm} \\
u_{0}^{ \pm} & 0
\end{array}\right) V=0
$$

Notice that the Green's function for $\mathcal{L}^{-1}$ is $G(x)=\frac{1}{2 \beta} e^{-|x| / \beta}$. Therefore

$$
\begin{aligned}
V(x) & =-G(x) *\left[\left(\begin{array}{cc}
\eta_{0} & u_{0}^{ \pm} \\
u_{0}^{ \pm} & 0
\end{array}\right) V\right](x) \\
& =-\int_{\mathbb{R}} G(x-y)\left(\begin{array}{cc}
\eta_{0}(y) & u_{0}^{ \pm}(y) \\
u_{0}^{ \pm}(y) & 0
\end{array}\right) V(y) d y \\
& =-\frac{1}{G(x)} \int_{\mathbb{R}} \frac{G(x-y) G(y)}{G(x)} \frac{1}{G(y)}\left(\begin{array}{cc}
\eta_{0}(y) & u_{0}^{ \pm}(y) \\
u_{0}^{ \pm}(y) & 0
\end{array}\right) V(y) d y .
\end{aligned}
$$

Since

$$
\left|\frac{G(x-y) G(y)}{G(x)}\right| \lesssim 1, \quad\left|\frac{\eta_{0}(y)}{G(y)}\right|+\left|\frac{u_{0}^{ \pm}(y)}{G(y)}\right| \lesssim 1,
$$

we conclude that $V$ decays exponentially

$$
|G(x) V(x)| \lesssim 1
$$


Expanding (2.8) into a $4 \times 4$ first order ODE system and checking the asymptotics we find that there are only two bounded solution branches, and they have the asymptotic behavior

$$
e^{-|x| / \beta} \quad \text { and } \quad|x| e^{-|x| / \beta} \quad \text { as } \quad|x| \rightarrow \infty .
$$

Together with (2.9) we know that the space of bounded solutions to (2.8) is at most one-dimensional. Recalling from the translation invariance that

$$
\mathscr{F}_{U}\left(U_{0}^{ \pm}, 0\right)\left[\left(U_{0}^{ \pm}\right)^{\prime}\right]=0,
$$

it follows that $\left(U_{0}^{ \pm}\right)^{\prime}$ is the only bounded solution to (2.8). Finally the parity condition yields the desired result.

The spectral property of $\mathscr{F}_{U}\left(U_{0}^{ \pm}, 0\right)$ given by Lemma 2.2 allows a use of the Implicit Function Theorem. Notice that for if $(u, \eta, \lambda)$ is a solution to (2.6) with $\lambda>0$, then so is $(-u, \eta,-\lambda)$. In fact this corresponds to the same wave propagating in the opposite direction. Therefore in the following analysis we will only consider the case $\lambda>0$.

Theorem 2.1 (Nearly stationary waves). For any $\beta \in \mathbb{R}$ there exists some positive $\lambda_{0}>0$ and $a C^{0}$ solution curve

$$
\mathscr{C}_{\text {loc }}^{\text {slow }}=\left\{\left(u^{ \pm}(\lambda), \eta(\lambda), \lambda\right): 0 \leq \lambda<\lambda_{0}\right\} \subset \mathscr{X} \times \mathbb{R}
$$

to problem (2.6) with the property that

$$
\begin{aligned}
& u(\lambda)=u_{0}^{+}+O(\lambda), \quad \eta(\lambda)=\eta_{0}+O(\lambda) \quad \text { in } \mathscr{X}, \\
& u(\lambda)>0 \quad \text { and } \quad \eta(\lambda)<0,
\end{aligned}
$$

where $\left(u_{0}^{+}, \eta_{0}\right)$ is given in (2.4).

Proof. The proof of the existence and uniqueness of the solution curves and (2.10) follows from Lemma 2.2 and a direct application of the Implicit Function Theorem.

Applying the maximum principle to the second equation of (2.6) we see that $\lambda u \geq \eta$. From (2.6) we also have

$$
-\beta^{2}\left[1-\lambda^{2}\left(1+\frac{1}{3 \beta^{2}}\right)\right] \eta^{\prime \prime}+\left(1-\lambda^{2}-\lambda u\right) \eta+\frac{1}{2} u^{2}=0 .
$$

From (2.10) we know that for $\lambda$ sufficiently small $1-\lambda^{2}-\lambda u>0$. Therefore, from the maximum principle we conclude that $\eta \leq 0$. If there is an $x_{1}$ such that $\eta\left(x_{1}\right)=$ $0=\max \eta$, then we have $\eta^{\prime}\left(x_{0}\right)=0$. Substituting this into the above equation leads to $\eta^{\prime \prime}\left(x_{0}\right)=u\left(x_{0}\right)=0$. Hence $(\eta-\lambda u)\left(x_{0}\right)=0$. Since $\eta-\lambda u \leq 0$, we see that $(\eta-\lambda u)\left(x_{0}\right)=\max (\eta-\lambda u)$, and thus $u^{\prime}\left(x_{0}\right)=0$. The uniqueness of ODE then implies that $(\eta, u) \equiv 0$, a contradiction. Therefore we must have

$$
\eta<0 \text {. }
$$

Direct calculation yields the equation for $u$ as

$$
\begin{aligned}
-\beta^{2}\left[1-\lambda^{2}\left(1+\frac{1}{3 \beta^{2}}\right)\right] u^{\prime \prime} & +\left[1-\lambda^{2}\left(1+\frac{1}{3 \beta^{2}}\right)+\frac{\lambda}{2}\left(1+\frac{1}{3 \beta^{2}}\right) u\right] u \\
+ & \left(\frac{\lambda}{3 \beta^{2}}+u\right) \eta=0 .
\end{aligned}
$$


From (2.10) and (2.4) we know that for any $\varepsilon>0$ there exist $\lambda>0$ sufficient small and $R_{0}>0$ sufficiently large such that

$$
\begin{aligned}
& \left\|u-u_{0}^{+}\right\|_{C^{2}(\mathbb{R})}+\left\|\eta-\eta_{0}\right\|_{C^{2}(\mathbb{R})}+\left\|u_{0}^{+}\right\|_{C^{0}\left(|x| \geq R_{0}\right)}+\left\|\eta_{0}\right\|_{C^{0}\left(|x| \geq R_{0}\right)}<\varepsilon, \\
& u>0 \quad \text { for }|x|<R_{0} .
\end{aligned}
$$

If inf $u<-\frac{\lambda}{3 \beta^{2}}<0$, then from the above equation we know that there exists some $x_{0}>R_{0}$ such that $u\left(x_{0}\right)=\inf u$. Continuity then yields the existence of $x_{1}$ with $x_{1}>R_{0}$ and $u\left(x_{1}\right)=0$ such that

$$
x_{1}=\min \{x>0: u(x)=0\} .
$$

Rewriting (2.13) as

$$
-\beta^{2}\left[1-\lambda^{2}\left(1+\frac{1}{3 \beta^{2}}\right)\right] u^{\prime \prime}+\left[1-\lambda^{2}\left(1+\frac{1}{3 \beta^{2}}\right)+\eta+\frac{\lambda}{2}\left(1+\frac{1}{3 \beta^{2}}\right) u\right] u+\frac{\lambda}{3 \beta^{2}} \eta=0,
$$

we see from (2.14) that $|\eta|<2 \varepsilon$ on $\left[x_{1},+\infty\right)$. Thus for $\lambda$ and $\varepsilon$ sufficiently small, applying the maximum principle on $\left[x_{1},+\infty\right)$ yields that

$$
u \geq 0 \quad \text { on }\left[x_{1},+\infty\right),
$$

which is a contradiction.

Therefore

$$
\inf u \geq-\frac{\lambda}{3 \beta^{2}} .
$$

Substituting this into (2.13), from the maximum principle we can infer that $u>0$, which is (2.11).

To investigate further the qualitative properties of the solutions, let us first recall the following result of [13, Theorem 2] on weakly coupled elliptic systems.

Theorem 2.2 ([13]). If $(u, v)$ is a classical solution to the following elliptic system

$$
\left\{\begin{array}{rll}
\Delta u+g(u, v)=0 & \text { in } & \mathbb{R}^{n}, \\
\Delta v+f(u, v)=0 & \text { in } & \mathbb{R}^{n}, \\
u, v>0 & \text { in } & \mathbb{R}^{n}, \\
u(x), v(x) \rightarrow 0 & \text { as } & |x| \rightarrow \infty,
\end{array}\right.
$$

where $f, g \in C^{1}([0, \infty) \times[0, \infty), \mathbb{R})$. Suppose further that

(i) $\frac{\partial g}{\partial v}, \frac{\partial f}{\partial u}$ are non-negative on $[0, \infty) \times[0, \infty)$; (quasi-monotonicity)

(ii) $\frac{\partial g}{\partial u}(0,0)<0$ and $\frac{\partial f}{\partial v}(0,0)<0$;

(iii) $\operatorname{det} A>0$, where

$$
A:=\left(\begin{array}{ll}
\frac{\partial g}{\partial u} & \frac{\partial g}{\partial v} \\
\frac{\partial f}{\partial u} & \frac{\partial f}{\partial v}
\end{array}\right)(0,0)
$$


Then there exist points $x_{0}, x_{1} \in \mathbb{R}^{n}$ such that $u(x)=u\left(\left|x-x_{0}\right|\right)$ and $v(x)=v\left(\left|x-x_{1}\right|\right)$. Moreover

$$
\frac{d u}{d r_{0}}<0 \quad \text { and } \quad \frac{d v}{d r_{1}}<0
$$

where $r_{0}:=\left|x-x_{0}\right|$ and $r_{1}:=\left|x-x_{1}\right|$.

From the above theorem we immediately obtain

Lemma 2.3 (Local monotonicity). Fix $\beta \in \mathbb{R}$. There exists $\lambda_{0}>0$ such that every solution $(u, \eta, \lambda) \in \mathscr{C}_{\text {loc }}^{\text {slow }}$ with $0 \leq \lambda<\lambda_{0}$ is strictly monotone in that for $x>0$,

$$
u^{\prime}<0 \quad \text { and } \quad \eta^{\prime}>0 .
$$

Proof. We see that $(u, \eta)$ satisfies equations (2.13) and (2.12). Setting $v:=-\eta$ and putting it into the form as in Theorem 2.2 we find that

$$
\begin{aligned}
& g(u, v)=-\frac{1}{\beta^{2}} u+\frac{\lambda}{3 \beta^{4} B} v+\frac{1}{\beta^{2} B} u v-\frac{\lambda}{2 \beta^{2} B}\left(1+\frac{1}{3 \beta^{2}}\right) u^{2}, \\
& f(u, v)=-\frac{1-\lambda^{2}}{\beta^{2} B} v+\frac{\lambda}{\beta^{2} B} u v+\frac{1}{2 \beta^{2} B} u^{2},
\end{aligned}
$$

where $B:=\left[1-\lambda^{2}\left(1+\frac{1}{3 \beta^{2}}\right)\right]>0$ for small $\lambda$. Direct computation shows that

$$
\left(\begin{array}{ll}
\frac{\partial g}{\partial u} & \frac{\partial g}{\partial v} \\
\frac{\partial f}{\partial u} & \frac{\partial f}{\partial v}
\end{array}\right)=\left(\begin{array}{cc}
-\frac{1}{\beta^{2}}+\frac{1}{\beta^{2} B} v-\frac{\lambda}{\beta^{2} B}\left(1+\frac{1}{3 \beta^{2}}\right) u & \frac{\lambda}{3 \beta^{4} B}+\frac{1}{\beta^{2} B} u \\
\frac{\lambda}{\beta^{2} B} v+\frac{1}{\beta^{2} B} u & -\frac{1-\lambda^{2}}{\beta^{2} B}+\frac{\lambda}{\beta^{2} B} u
\end{array}\right)
$$

From Theorem 2.1 we know that $u, v>0$ when $\lambda$ is small, which implies that (i) (iii) of Theorem 2.2 are satisfied. Therefore (2.16) holds.

Another application of Theorem 2.2 to the local solution near the bifurcation point $\left(u_{0}^{+}, \eta_{0}, 0\right)$ is the following result on the local uniqueness of the solution curve $\mathscr{C}_{\text {loc }}^{\text {slow }}$. In particular this result shows that all classical solutions near $\left(u_{0}^{+}, \eta_{0}, 0\right)$ with $\lambda>0$ must be even and monotone on the positive axis.

Corollary 2.3 (Local uniqueness). Denote by $\mathcal{B}_{r}$ the ball of radius $r>0$ in $\left(C^{2}(\mathbb{R}) \cap C_{0}(\mathbb{R})\right) \times$ $\left(C^{2}(\mathbb{R}) \cap C_{0}(\mathbb{R})\right) \times \mathbb{R}$ centered at $\left(u_{0}^{+}, \eta_{0}, 0\right)$. There exists $\varepsilon>0$ such that for $\lambda>0$,

$$
\mathscr{F}^{-1}(0) \cap \mathcal{B}_{\varepsilon}=\mathscr{C}_{\text {loc }}^{\text {slow }} \cap \mathcal{B}_{\varepsilon} .
$$

Proof. Consider a solution $(u, \eta, \lambda)$ to equations (2.12)-(2.13) with

$$
\left\|u-u_{0}^{+}\right\|_{C^{2}(\mathbb{R})}+\left\|\eta-\eta_{0}\right\|_{C^{2}(\mathbb{R})}+|\lambda|<\varepsilon .
$$

There exists an $R_{0}>0$ large enough such that

$$
\begin{aligned}
& \left\|u-u_{0}^{+}\right\|_{C^{2}(\mathbb{R})}+\left\|\eta-\eta_{0}\right\|_{C^{2}(\mathbb{R})}+|\lambda|+\left\|u_{0}^{+}\right\|_{C^{0}\left(|x| \geq R_{0}\right)}+\left\|\eta_{0}\right\|_{C^{0}\left(|x| \geq R_{0}\right)}<\varepsilon, \\
& u>0, \quad \eta<0, \quad u^{\prime}<0, \quad \eta^{\prime}>0 \quad \text { for } \quad|x|<R_{0} .
\end{aligned}
$$


Hence if $\sup \eta>0$, then from continuity there exists $x_{0}:=\min \{x>0: \eta(x)=0\}$ such that $\eta\left(x_{0}\right)=0$ and $x_{0}>R_{0}$. From (2.18) we see that

$$
1-\lambda^{2}-\lambda u>0 \quad \text { on }\left[x_{0},+\infty\right) \text {. }
$$

Applying the maximum principle to (2.12) on $\left[x_{0},+\infty\right)$ yields that $\eta \leq 0$ on $\left[x_{0},+\infty\right)$. Together with (2.18), this fact contradicts the assumption that $\sup \eta>0$. Therefore we must have $\eta \leq 0$.

In a similar way if inf $u<0$, then we may find $x_{1}:=\min \{x>0: u(x)=0\}$ such that $u\left(x_{0}\right)=0$ and $x_{0}>R_{0}$. The maximum principle applied to (2.15) on $\left[x_{1},+\infty\right)$ leads to the conclusion that $u \geq 0$, contradicting to the assumption that inf $u<0$. Thus $u \geq 0$.

If there exists some $x_{0} \geq 0$ such that $\eta\left(x_{0}\right)=0$, then $\eta\left(x_{0}\right)=\sup \eta$, and hence $\eta^{\prime}\left(x_{0}\right)=0$ and $\eta^{\prime \prime}\left(x_{0}\right) \leq 0$. From (2.12) we find that $u\left(x_{0}\right)=0$. This also means that $u\left(x_{0}\right)=\inf u$, and so $u^{\prime}\left(x_{0}\right)=0$. Uniqueness of the ODE then implies that $\eta=u \equiv 0$, which contradicts (2.18). The same argument applies to the situation if $u$ touches zero at some finite point.

The above argument indicates that for any small $(u, \eta, \lambda) \in \mathscr{F}^{-1}(0) \cap \mathcal{B}_{\varepsilon}$,

$$
u>0 \quad \text { and } \quad \eta<0 .
$$

Then for $\lambda>0$ one may apply Theorem 2.2 to conclude that $u$ and $\eta$ are both even. Therefore the uniqueness of $\mathscr{C}_{\text {loc }}^{\text {slow }}$ within $\mathscr{F}^{-1}(0) \cap\left(\mathscr{X} \times \mathbb{R}^{+}\right)$gives (2.17).

2.3. Nodal pattern. Now for each fixed $\beta \in \mathbb{R}$ we introduce the set

$$
\mathcal{O}:=\left\{(u, \eta, \lambda) \in \mathscr{X} \times \mathbb{R}^{+}: 1-\lambda^{2}\left(1+\frac{1}{3 \beta^{2}}\right)>0\right\} .
$$

The results of Theorem 2.1 and Lemma 2.3 naturally suggest us to consider the following "nodal properties"

$$
\begin{array}{ll}
u>0 & \text { in } \mathbb{R}, \\
\eta<0 & \text { in } \mathbb{R}, \\
u^{\prime}<0 & \text { in } \mathbb{R}^{+}, \\
\eta^{\prime}>0 & \text { in } \mathbb{R}^{+} .
\end{array}
$$

Lemma 2.4 (Open property). Let $\left(u_{*}, \eta_{*}, \lambda_{*}\right) \in \mathcal{O} \cap \mathscr{F}^{-1}(0)$ be given and suppose that it satisfies (2.20). There exists $\varepsilon=\varepsilon\left(u_{*}, \eta_{*}, \lambda_{*}\right)>0$ such that, if $(u, \eta, \lambda) \in \mathcal{O} \cap \mathscr{F}^{-1}(0)$ and

$$
\left\|u-u_{*}\right\|_{C^{2}(\mathbb{R})}+\left\|\eta-\eta_{*}\right\|_{C^{2}(\mathbb{R})}+\left|\lambda-\lambda_{*}\right|<\varepsilon,
$$

then $(u, \eta, \lambda)$ also satisfies (2.20).

Proof. The proof of (2.20a ) and (2.20b) follows the same argument as in the proof of Corollary 2.3 by replacing $\left(u_{0}^{+}, \eta_{0}, 0\right)$ with $\left(u_{*}, \eta_{*}, \lambda_{*}\right)$. The proof for (2.20c)-(2.20d) then follows directly from the application of Theorem 2.2 .

Lemma 2.5 (Closed property). Let $\left\{\left(u_{n}, \eta_{n}, \lambda_{n}\right)\right\} \subset \mathcal{O} \cap \mathscr{F}^{-1}(0)$ be given and suppose that $\left(u_{n}, \eta_{n}, \lambda_{n}\right) \rightarrow(u, \eta, \lambda) \in \mathcal{O} \cap \mathscr{F}^{-1}(0)$ in $C^{2}(\mathbb{R}) \times C^{2}(\mathbb{R}) \times \mathbb{R}$. If each $\left(u_{n}, \eta_{n}, \lambda_{n}\right)$ satisfies (2.20), then $(u, \eta, \lambda)$ also satisfies (2.20) unless $u=\eta \equiv 0$. 
Proof. First we see that

$$
\begin{array}{lll}
u \geq 0, & \eta \leq 0, & \lambda \geq 0, \quad \text { and } \\
u^{\prime} \leq 0, & \eta^{\prime} \geq 0 & \text { in } \mathbb{R}^{+} .
\end{array}
$$

If there exists $x_{0}$ such that $u\left(x_{0}\right)=0$, then $u\left(x_{0}\right)=\inf u$, and hence $u^{\prime}\left(x_{0}\right)=0$. From the equation (2.13) and maximum principle we see that $\eta\left(x_{0}\right)=0$. Therefore $\eta\left(x_{0}\right)=\sup \eta$. So $\eta^{\prime}\left(x_{0}\right)=0$. Thus from the uniqueness of ODE we know that $u=\eta \equiv 0$.

Lemma 2.6 (Nodal property). If $\mathcal{K}$ is any connected subset of $\mathcal{O} \mathscr{F}^{-1}(0)$ that contains $\mathscr{C}_{\text {loc }}^{\text {slow }}$, then every $(u, \eta, \lambda) \in \mathcal{K}$ exhibits (2.20).

Proof. First note that each $(u(\lambda), \eta(\lambda), \lambda) \in \mathscr{C}_{\text {loc }}^{\text {slow }}$ satisfies (2.20). Recall the definition of $\mathcal{B}_{r}$ in Corollary 2.3. Fix $0<\lambda<\lambda_{0}$ and take $\varepsilon$ to be sufficiently small, the local uniqueness of $\mathscr{C}_{\text {loc }}^{\text {slow implies that }}$

$$
\mathcal{K} \cap \mathcal{B}_{\varepsilon}=\mathscr{C}_{\text {loc }}^{\text {slow }} \cap \mathcal{B}_{\varepsilon},
$$

and $\mathcal{K} \backslash \mathcal{B}_{\varepsilon}$ is the connected component containing $(u(\lambda), \eta(\lambda), \lambda)$. Applying Lemmas 2.4 and 2.5 completes the proof.

2.4. Monotone fronts. Next we define the concept of monotone fronts.

Definition 2.4. For $\lambda>0$ and $\lambda^{2}\left(1+\frac{1}{3 \beta^{2}}\right)<1$, we say $(u, \eta, \lambda)$ is a monotone front solution of (2.6) if $(u, \eta) \in C_{\mathrm{b}}^{2}(\mathbb{R}) \times C_{\mathrm{b}}^{2}(\mathbb{R})$, and

$$
\lim _{x \rightarrow+\infty}(u(x), \eta(x))=(0,0), \quad \text { and } \quad u>0, \quad \eta<0, \quad u^{\prime} \leq 0, \quad \eta^{\prime} \geq 0 \quad \text { in } \mathbb{R},
$$

where $C_{\mathrm{b}}^{2}(\mathbb{R})$ is the set of $C^{2}$ functions with bounded norms.

Lemma 2.7 (Nonexistence of monotone fronts). There exists some $\beta_{0}>0$ such that if $\lambda>0$ and $|\beta|<\beta_{0}$, then system (2.6) does not admit any monotone front solution in the sense of (2.22).

Proof. Suppose $(u, \eta)$ is a monotone front solution to (2.6). Then since $u, \eta$ are bounded and monotone,

$$
(\bar{u}, \bar{\eta}):=\lim _{x \rightarrow-\infty}(u(x), \eta(x))
$$

exists, and $\bar{u}>0, \bar{\eta}<0$. Evaluating (2.12) at $-\infty$ leads to

$$
\bar{u}<\frac{1-\lambda^{2}}{\lambda}, \quad \text { and } \quad \bar{\eta}=-\frac{\bar{u}^{2}}{2\left(1-\lambda^{2}-\lambda \bar{u}\right)} .
$$

Substituting the above equation into (2.13) and evaluating the equation at $-\infty$ yields

$$
-(2-B) \bar{u}^{2}-\lambda B \bar{u}+2\left(1-\lambda^{2}\right) B=0,
$$

where $B=1-\lambda^{2}\left(1+\frac{1}{3 \beta^{2}}\right) \in(0,1)$. Solving this quadratic equation together with the constraint that $\bar{u}>0$ yields

$$
\bar{u}=\frac{\sqrt{\lambda^{2} B^{2}+8 B(2-B)\left(1-\lambda^{2}\right)}-\lambda B}{2(2-B)} .
$$


On the other hand, multiplying (2.12) by $\eta^{\prime}$ and multiplying (2.13) by $u^{\prime}$ and summing up, it follows that

$$
\begin{aligned}
{\left[-\frac{\beta^{2} B}{2}\left(\left(u^{\prime}\right)^{2}+\left(\eta^{\prime}\right)^{2}\right)+\right.} & \left.\frac{1-\lambda^{2}}{2} \eta^{2}+\frac{B}{2} u^{2}+\frac{\lambda}{6}\left(1+\frac{1}{3 \beta^{2}}\right) u^{3}+\frac{1}{2} u^{2} \eta\right]^{\prime} \\
& -\lambda u \eta \eta^{\prime}+\frac{\lambda}{3 \beta^{2}} \eta u^{\prime}=0 .
\end{aligned}
$$

We can rewrite the last two terms above as

$$
-\lambda u \eta \eta^{\prime}+\frac{\lambda}{3 \beta^{2}} \eta u^{\prime}=\left(-\frac{\lambda}{2} u \eta^{2}+\frac{\lambda}{3 \beta^{2}} \eta u\right)^{\prime}+\frac{\lambda}{2} u^{\prime} \eta^{2}-\frac{\lambda}{3 \beta^{2}} \eta^{\prime} u .
$$

The definition of monotone front implies that $\frac{\lambda}{2} u^{\prime} \eta^{2}-\frac{\lambda}{3 \beta^{2}} \eta^{\prime} u \leq 0$, and hence we have

$$
\frac{1-\lambda^{2}}{2} \bar{\eta}^{2}+\frac{B}{2} \bar{u}^{2}+\frac{\lambda}{6}\left(1+\frac{1}{3 \beta^{2}}\right) \bar{u}^{3}+\frac{1}{2} \bar{u}^{2} \bar{\eta}-\frac{\lambda}{2} \bar{u} \bar{\eta}^{2}+\frac{\lambda}{3 \beta^{2}} \bar{u} \bar{\eta} \leq 0 .
$$

Recalling (2.23) and the definition of $B$ the above inequality can be simplified to

$$
\bar{u}\left(\frac{1}{4} \bar{\eta}+\frac{B}{2}+\frac{1-B}{6 \lambda} \bar{u}\right)+\frac{\lambda}{3 \beta^{2}} \bar{\eta} \leq 0,
$$

which further leads to

$$
\bar{u}\left(\frac{B}{2}+\frac{1-B}{6 \lambda} \bar{u}\right)-\left(\frac{1}{4} \bar{u}+\frac{1-B-\lambda^{2}}{\lambda}\right) \frac{\bar{u}^{2}}{2\left(1-\lambda^{2}-\lambda \bar{u}\right)} \leq 0 .
$$

Solving above yields

$$
\bar{u} \geq \frac{2 \sqrt{4(1-B)^{2}\left(1-\lambda^{2}\right)^{2}+3(7-4 B) B \lambda^{2}\left(1-\lambda^{2}\right)}-4(1-B)\left(1-\lambda^{2}\right)}{\lambda(7-4 B)} .
$$

Combining this with (2.24) and explicitly solving the resulting inequality leads to

$$
G\left(\lambda^{2}, t\right) \geq 0
$$

where $t=1+\frac{1}{3 \beta^{2}}>1$ and

$$
G(z, t):=\left(-20+\frac{13}{t}\right) z^{3}+\left(-60+\frac{33}{t}+32 t\right) z^{2}+\left(-39+\frac{18}{t}+32 t\right) z-9 .
$$

Recall from Definition 2.4 that we are only interested in the interval $z \in\left(0,1 / t^{2}\right)$. It is easy to see that

$$
G_{z}(0, t), G_{z z}(0, t)>0 .
$$

Looking at $G(z, t)$, we find that for $t>t_{1}$ sufficiently large, say $t_{1} \approx 2.264$, we have

$$
G\left(\frac{1}{t^{2}}, t\right)<0 .
$$

For a fixed $t>1$, solving a quartic inequality it follows that

$$
G_{z z}\left(\frac{1}{t^{2}}, t\right)>0
$$


when $t>t_{2}$ for some large enough $t_{2}$ (for example $t_{2} \approx 1.68$ ). This together with (2.27) and the fact that $G_{z z}(z, t)$ is linear in $z$ implies that for $t>t_{2}, G_{z z}(z, t)>0$ for $0<z<\frac{1}{t^{2}}$. Therefore we have

$$
G_{z}(z, t)>G_{z}(0, t)>0 \quad \text { for } \quad 0<z<\frac{1}{t^{2}} .
$$

So for $t>\max \left\{t_{1}, t_{2}\right\}$, corresponding to $\beta^{2}<\beta_{0}^{2}$ with

$$
\beta_{0}^{2}=\min \left\{\frac{1}{3 t_{1}-1}, \frac{1}{3 t_{2}-1}\right\}
$$

it yields that

$$
G(z, t)<0 \quad \text { for } \quad 0<z<\frac{1}{t^{2}}
$$

which contradicts (2.26). This completes the proof of the lemma.

Remark 2.1. Taking $t_{1} \approx 2.264$ and $t_{2} \approx 1.68$, we may choose $\beta_{0} \approx 0.5$.

2.5. Global continuation. Now that we have obtained the local bifurcation result, we will extend the local solution curves constructed in Section 2.2 to the non-perturbative regime using a global implicit function theorem developed in [20].

Theorem 2.5. There exists a curve $\mathscr{C}^{\text {slow }}$ containing $\mathscr{C}_{\text {loc }}^{\text {slow }}$, which admits a global $C^{0}$ parametrization

$$
\mathscr{C}^{\text {slow }}:=\{(u(s), \eta(s), \lambda(s)): s \in(0, \infty)\} \subset \mathcal{O} \cap \mathscr{F}^{-1}(0)
$$

with $\lim _{s \searrow 0}(u(s), \eta(s), \lambda(s))=\left(u_{0}^{+}, \eta_{0}, 0\right)$ and satisfies the following.

(a) At each $s \in(0, \infty)$, the linearized operator $\mathscr{F}_{(u, \eta)}(u(s), \eta(s), \lambda(s)): \mathscr{X} \times \mathbb{R}^{+} \rightarrow$ $\mathscr{Y}$ is Fredholm index 0.

(b) One of the following alternatives holds as $s \rightarrow \infty$.

(A1) (Blowup) The quantity

$N(s):=\|(u(s), \eta(s))\|_{\mathscr{X}}+\lambda(s)+\frac{1}{\operatorname{dist}((u(s), \eta(s), \lambda(s)), \partial \mathcal{O})} \rightarrow \infty$.

(A2) (Loss of compactness) There exists a sequence $s_{n} \rightarrow \infty$ with $\sup _{n} N\left(s_{n}\right)<$ $\infty$, but $\left(u^{+}\left(s_{n}\right), \eta\left(s_{n}\right), \lambda\left(s_{n}\right)\right)$ has no convergent subsequence in $\mathscr{X} \times \mathbb{R}^{+}$.

(A3) (Loss of Fredholmness) There exists a sequence $s_{n} \rightarrow \infty$ with $\sup _{n} N\left(s_{n}\right)<$ $\infty$ and so that $\left(u\left(s_{n}\right), \eta\left(s_{n}\right), \lambda\left(s_{n}\right)\right) \rightarrow\left(u_{*}, \eta_{*}, \lambda_{*}\right)$ in $\mathscr{X} \times \mathbb{R}^{+}$, however $\mathscr{F}_{(u, \eta)}\left(u_{*}, \eta_{*}, \lambda_{*}\right)$ is not Fredholm index 0.

(A4) (Closed loop) There exists $T>0$ such that $(u(s+T), \eta(s+T), \lambda(s+T))=$ $(u(s), \eta(s), \lambda(s))$ for all $s \in(0, \infty)$.

(c) Near each point $\left(u\left(s_{0}\right), \eta\left(s_{0}\right), \lambda\left(s_{0}\right)\right) \in \mathscr{C}^{\text {slow }}$, we can locally reparameterize $\mathscr{C}^{\text {slow }}$ so that $s \mapsto(u(s), \eta(s), \lambda(s))$ is real analytic.

Proof. The proof follows from [20, Theorem B.1] and [19, Theorem 6.1], since from Lemma 2.2 we know that $\mathscr{F}_{(u, \eta)}\left(u_{0}, \eta_{0}, \lambda_{0}\right): \mathscr{X} \times \mathbb{R}^{+} \rightarrow \mathscr{Y}$ is an isomorphism.

Given a $(u, \eta, \lambda) \in \mathscr{X} \times \mathbb{R}$, direct computation yields that the linearized operator

$$
\mathscr{F}_{(u, \eta)}(u, \eta, \lambda)=\left(\begin{array}{cc}
\mathcal{L}+\eta & -\lambda\left(1+\frac{1}{3 \beta^{2}}\right) \\
-\lambda \mathcal{L}+u & \mathcal{L}+\left(\frac{\lambda}{3 \beta^{2}}+u\right)
\end{array}\right): \mathscr{X} \rightarrow \mathscr{Y}
$$


Since $(u, \eta) \in \mathscr{X}$, the limiting operator as $|x| \rightarrow \infty$ is

$$
\tilde{\mathscr{F}}_{(u, \eta)}(u, \eta, \lambda):=\left(\begin{array}{cc}
\mathcal{L} & -\lambda\left(1+\frac{1}{3 \beta^{2}}\right) \mathcal{L}+\frac{\lambda}{3 \beta^{2}} \\
-\lambda \mathcal{L} & \mathcal{L}
\end{array}\right): \mathscr{X} \rightarrow \mathscr{Y} .
$$

Lemma 2.8. For $(u, \eta, \lambda) \in \mathcal{O}$, the limiting operator $\tilde{\mathscr{F}}_{(u, \eta)}(u, \eta, \lambda)$ is invertible.

Proof. If $V=(v, \zeta) \in \mathscr{X}$ such that $\tilde{\mathscr{F}}_{(u, \eta)}(u, \eta, \lambda)[V]=0$, then a row elimination yields

$$
\left[1-\lambda^{2}\left(1+\frac{1}{3 \beta^{2}}\right)\right] \mathcal{L} \zeta+\frac{\lambda^{2}}{3 \beta^{2}} \zeta=0 .
$$

Thus $\zeta=0$, which also implies that $\mathcal{L} v=0$, and hence $v=0$. This shows that $\tilde{\mathscr{F}}_{(u, \eta)}(u, \eta, \lambda)$ is injective.

Now for any $f=\left(f_{1}, f_{2}\right) \in \mathscr{Y}$, consider solving $\tilde{\mathscr{F}}_{(u, \eta)}(u, \eta, \lambda)[V]=f$ for $V \in \mathscr{X}$. By a similar argument as before, we can perform a row elimination to solve for $\zeta$ from

$$
\left[1-\lambda^{2}\left(1+\frac{1}{3 \beta^{2}}\right)\right] \mathcal{L} \zeta+\frac{\lambda^{2}}{3 \beta^{2}} \zeta=\lambda f_{1}+f_{2},
$$

and then plug this back to the system to solve for $v$. This way we verify that $\tilde{\mathscr{F}}_{(u, \eta)}(u, \eta, \lambda)$ is also surjective. Therefore the conclusion follows.

With the help of Lemma 2.8, we may follow the argument in [40, 19, to prove that $\mathscr{F}_{(u, \eta)}(u, \eta, \lambda): \mathscr{X} \rightarrow \mathscr{Y}$ is locally proper. Finally we have

Lemma 2.9. For $(u, \eta, \lambda) \in \mathcal{O}$, the linearized operator $\mathscr{F}_{(u, \eta)}(u, \eta, \lambda)$ is Fredholm with index 0 .

Proof. The lemma can be proved by a homotopy argument. Consider the operator $L_{t}:=t \tilde{\mathscr{F}}_{(u, \eta)}(u, \eta, \lambda)+(1-t)\left(\mathscr{F}_{(u, \eta)}(u, \eta, \lambda)-\tilde{\mathscr{F}}_{(u, \eta)}(u, \eta, \lambda)\right)$ for $t \in[0,1]$. Thus for any $t \in[0,1]$ the limiting operator of $L_{t}$ is $\tilde{\mathscr{F}}_{(u, \eta)}(u, \eta, \lambda)$. The previous argument proves that $L_{t}$ is locally proper, and thus Fredholm. Hence by continuity of the index we see that

$$
\operatorname{ind} \mathscr{F}_{(u, \eta)}(u, \eta, \lambda)=\operatorname{ind} L_{1}=\operatorname{ind} \tilde{\mathscr{F}}_{(u, \eta)}(u, \eta, \lambda)=0,
$$

which completes the proof.

Now we have all needed properties to further winnow down the alternatives in Theorem 2.5. By Lemma 2.9, for any $(u(s), \eta(s), \lambda(s)) \in \mathscr{C}^{\text {slow }}, \mathscr{F}_{(u, \eta)}(u(s), \eta(s), \lambda(s)): \mathscr{X} \rightarrow$ $\mathscr{Y}$ is Fredholm index 0. Thus we know that (A3) does not occur.

The loop alternative (A4) can also be ruled out by the nodal property Lemma 2.6 combined with the uniqueness results Proposition 2.1] and Corollary 2.3 .

As for (A2), we may adapt [19, Lemma 6.3] in our current setting to give the following

Lemma 2.10 (Compactness or front). Suppose that $\left\{\left(u_{n}, \eta_{n}, \lambda_{n}\right)\right\} \subset \mathscr{F}^{-1}(0) \cap \mathcal{O}$ satisfies

$$
\sup _{n \geq 1}\left(\left\|\left(u_{n}, \eta_{n}\right)\right\|_{\mathscr{X}}+\frac{1}{\operatorname{dist}\left(\left(u_{n}, \eta_{n}, \lambda_{n}\right), \partial \mathcal{O}\right)}\right)<\infty,
$$

and each $\left(u_{n}, \eta_{n}\right)$ is strictly monotone in that $\partial_{x} u_{n}<0, \partial_{x} \eta_{n}>0$ for $x>0$. Then, either 
(i) (Compactness) $\left\{\left(u_{n}, \eta_{n}, \lambda_{n}\right)\right\}$ has a convergent subsequence in $\mathscr{X} \times \mathbb{R}$; or

(ii) (Monotone front) there exists a sequence of translations $x_{n} \rightarrow+\infty$ so that we can extract a convergent subsequence

$$
\left(u_{n}, \eta_{n}\right)\left(\cdot+x_{n}\right) \longrightarrow(u, \eta) \in C_{\mathrm{b}}^{2+\alpha}(\mathbb{R}) \quad \text { in } C_{\mathrm{loc}}^{2}(\mathbb{R}), \quad \lambda_{n} \longrightarrow \lambda,
$$

with $(u, \eta, \lambda) \in \mathcal{O}$. The limit is a monotone front solution of (2.6) in the sense of Definition 2.4.

Proof. Given the assumptions of the lemma, we know that up to a subsequence $\lambda_{n} \rightarrow \lambda$ with $1-\lambda^{2}\left(1+\frac{1}{3 \beta^{2}}\right)>0$. If $\left(u_{n}, \eta_{n}\right)$ is equi-decaying in the sense that for any $\varepsilon>0$ there exists some $R>0$ such that

$$
\sup _{n}\left\|\left(u_{n}, \eta_{n}\right)\right\|_{C^{2}((R, \infty))}<\varepsilon,
$$

then obviously $\left(u_{n}, \eta_{n}\right)$ has a convergent subsequence in $\mathscr{X}$, and hence leads to (i).

If $\left(u_{n}, \eta_{n}\right)$ is not equi-decaying, then there exists some $\varepsilon_{0}>0$ and a sequence $\left\{x_{n}\right\}$ with $x_{n} \rightarrow+\infty$ such that for all $n \geq 1$,

$$
\sup _{0 \leq i \leq 2}\left|\partial_{x}^{i}\left(u_{n}, \eta_{n}\right)\left(x_{n}\right)\right| \geq \varepsilon_{0} .
$$

Set $\left(v_{n}, \zeta_{n}\right):=\left(u_{n}, \eta_{n}\right)\left(\cdot+x_{n}\right)$. Since $\left(v_{n}, \zeta_{n}\right)$ is uniformly bounded in $\mathscr{X}$, there is a subsequence, still denoted by the same labeling, $\left(v_{n}, \zeta_{n}\right) \rightarrow(u, \eta) \in \mathscr{X}$ in $C_{\text {loc }}^{2}(\mathbb{R})$. Local convergence is enough to ensure that $\left(u^{+}, \eta\right)$ solves (2.6). The monotonicity of $\left(u_{n}, \eta_{n}\right)$ confirms that

$$
\partial_{x} u \leq 0, \quad \partial_{x} \eta \geq 0
$$

By definition of $\left(v_{n}, \zeta_{n}\right)$ we see that

$$
\left|\partial_{x}^{i}(u, \eta)(0)\right| \geq \varepsilon_{0} \quad \text { for some } i \leq 2 .
$$

Thus $(u, \eta) \not \equiv(0,0)$. The maximum principle then implies that $u>0$ and $\eta<0$.

Putting all of the above together, we finally arrive at our main result of this section.

Theorem 2.6 (Slow waves). For any $\beta$ with $|\beta|<\beta_{0}$ where $\beta_{0}$ is given in Lemma 2.7, the global curve $\mathscr{C}^{\text {slow }}$ constructed in Theorem 2.5 enjoys the following properties.

(a) (Symmetry and monotonicity) Each solution on $\mathscr{C}^{\text {slow }}$ is even and

$$
\begin{array}{rlrl}
\eta(s)<0 & u(s)>0 & & \text { on } \mathbb{R}, \\
\partial_{x} \eta(s)>0 & \partial_{x} u(s)<0 & \text { on } \mathbb{R}^{+} .
\end{array}
$$

(b) (Loss of ellipticity) Following $\mathscr{C}^{\text {slow }}$ to its extreme, the system loses ellipticity in that

$$
\lim _{s \rightarrow \infty} \lambda(s)=\left(1+\frac{1}{3 \beta^{2}}\right)^{-1 / 2} .
$$

Proof. Note that property (a) follows from the nodal properties Lemma 2.6. From the previous discussion, at the extreme of the solution curve, (A3) and (A4) cannot occur. Lemma 2.10 together with Lemma 2.7 rules out (A2), Therefore we are only left with blowup alternative. Since $\lambda$ is always bounded in $\mathcal{O}$, one can remove $\lambda(s)$ from the blowup quantity in (2.28). 
From the local uniqueness and the nodal properties we know that $\lim _{s \rightarrow \infty} \lambda(s)>0$. So if (2.30) is false, then there exists a sequence $\left\{s_{n}\right\}, s_{n} \rightarrow \infty$ with the corresponding solutions $\left(u_{n}, \eta_{n}, \lambda_{n}\right):=(u, \eta, \lambda)\left(s_{n}\right) \in \mathcal{O} \cap \mathscr{F}^{-1}(0)$ such that

$$
\lambda_{n} \rightarrow \lambda_{*}<\left(1+\frac{1}{3 \beta^{2}}\right)^{-1 / 2}, \quad\left\|\left(u_{n}, \eta_{n}\right)\right\|_{\mathscr{X}} \rightarrow \infty .
$$

Moreover $\lambda_{*}>0$. Since the system (2.6) is semi-linear, weakly coupled and has no first-order derivatives, elliptic regularity implies that $\left\|\left(u_{n}, \eta_{n}\right)\right\|_{C^{0}} \rightarrow \infty$. From (a), this is equivalent to

$$
u_{n}(0)-\eta_{n}(0) \rightarrow \infty
$$

From the second equation in (2.6) and the fact that $\left(\eta_{n}-\lambda_{n} u_{n}\right)(0)=\min \left(\eta_{n}-\lambda_{n} u_{n}\right)$, it follows that

$$
\frac{1}{2} u_{n}^{2}(0)+\left(\eta_{n}-\lambda_{n} u_{n}\right)(0)=\beta^{2}\left(\eta_{n}-\lambda_{n} u_{n}\right)^{\prime \prime}(0) \geq 0
$$

From this it must hold that

$$
u_{n}(0) \rightarrow \infty \text {. }
$$

Similarly, evaluating (2.13) at $x=0$ and using that $u_{n}^{\prime \prime}(0) \leq 0$ we find that

$$
\eta_{n}(0) \leq-\frac{1-\lambda_{n}^{2}\left(1+\frac{1}{3 \beta^{2}}\right)+\frac{\lambda_{n}}{2}\left(1+\frac{1}{3 \beta^{2}}\right) u_{n}(0)}{\frac{\lambda_{n}}{3 \beta^{2}}+u_{n}(0)} u_{n}(0) .
$$

For $n$ sufficiently large, from the above inequality, we have that

$$
\eta_{n}(0) \leq-\frac{2 \lambda_{n}}{5}\left(1+\frac{1}{3 \beta^{2}}\right) u_{n}(0) .
$$

Recall (2.25). Integrating the equation over $(0, \infty)$ we find that

$$
\frac{1-\lambda_{n}^{2}}{2} \eta_{n}^{2}(0)+\frac{1}{2} u_{n}^{2}(0)\left[B+\frac{\lambda_{n}}{3}\left(1+\frac{1}{3 \beta^{2}}\right) u_{n}(0)+\eta_{n}(0)\right]>0 .
$$

From (2.32) we obtain

$$
\frac{1-\lambda_{n}^{2}}{2} \eta_{n}^{2}(0)+\frac{1}{2} u_{n}^{2}(0)\left[B-\frac{\lambda_{n}}{15}\left(1+\frac{1}{3 \beta^{2}}\right) u_{n}(0)\right]>0 .
$$

Further using (2.6) we have that for any $\delta>0$,

$$
\begin{aligned}
& \mathcal{L}\{\delta\left.\lambda_{n}\left(1+\frac{1}{3 \beta^{2}}\right) \eta_{n}+\left[1-(1+\delta) \lambda_{n}^{2}\left(1+\frac{1}{3 \beta^{2}}\right)\right] u_{n}\right\} \\
&+\left(\frac{\lambda_{n}}{3 \beta^{2}}+u_{n}\right) \eta_{n}+\frac{1}{2}(1+\delta) \lambda_{n}\left(1+\frac{1}{3 \beta^{2}}\right) u_{n}^{2}=0 .
\end{aligned}
$$

From (2.31) there exists $\delta_{0}>0$ such that for $n$ sufficiently large,

$$
1-(1+\delta) \lambda_{n}^{2}\left(1+\frac{1}{3 \beta^{2}}\right)>0 \quad \text { for all } 0<\delta<\delta_{0} .
$$

From (2.33) we see that

$$
\left|\eta_{n}(0)\right|=O\left(\left|u_{n}(0)\right|^{3 / 2}\right) \quad \text { as } n \rightarrow \infty .
$$


Therefore for any $0<\delta<\delta_{0}$ there exists some $n_{0}$ large enough such that for $n \geq n_{0}$

$$
\delta \lambda_{n}\left(1+\frac{1}{3 \beta^{2}}\right) \eta_{n}(0)+\left[1-(1+\delta) \lambda_{n}^{2}\left(1+\frac{1}{3 \beta^{2}}\right)\right] u_{n}(0)<0 .
$$

Denote by $x_{n} \in[0, \infty)$ the point where $\delta \lambda_{n}\left(1+\frac{1}{3 \beta^{2}}\right) \eta_{n}+\left[1-(1+\delta) \lambda_{n}^{2}\left(1+\frac{1}{3 \beta^{2}}\right)\right] u_{n}$ achieves its minimum. Then it holds that $\eta_{n}(0) \leq \eta_{n}\left(x_{n}\right)<0$, and

$\delta \lambda_{n}\left(1+\frac{1}{3 \beta^{2}}\right) \eta_{n}\left(x_{n}\right) \leq \delta \lambda_{n}\left(1+\frac{1}{3 \beta^{2}}\right) \eta_{n}(0)+\left[1-(1+\delta) \lambda_{n}^{2}\left(1+\frac{1}{3 \beta^{2}}\right)\right]\left[u_{n}(0)-u_{n}\left(x_{n}\right)\right]$.

From this we conclude that

$$
\left|\eta_{n}\left(x_{n}\right)\right|=O\left(\left|u_{n}(0)\right|^{3 / 2}\right) \quad \text { as } n \rightarrow \infty .
$$

Evaluating equation (2.34) at $x_{n}$ indicates that

$$
\left(\frac{\lambda_{n}}{3 \beta^{2}}+u_{n}\left(x_{n}\right)\right) \eta_{n}\left(x_{n}\right)+\frac{1}{2}(1+\delta) \lambda_{n}\left(1+\frac{1}{3 \beta^{2}}\right) u_{n}^{2}\left(x_{n}\right)>0
$$

which contradicts the asymptotics (2.35).

\section{Bifurcation From Classical Boussinesq SupercritiCAL WAVES}

In this section we focus on fast traveling solitary waves with wave speed $\lambda>1$. Different from the previous section, here we will consider the wave speed as given, and restrict the four parameters $(a, b, c, d)$ on a one-parameter curve to perform the bifurcation. The base point of the bifurcation corresponds to the solution to the classical Boussinesq system which has $a=b=c=0$ and $d=\frac{1}{3}$ in (1.1) (see, for example, [3, 11, 30, 35]). As is discussed in [15], the solitary waves $\left(u_{f}, \eta_{f}\right)$ satisfy

$$
\left\{\begin{array}{l}
\left(u_{f}^{\prime}\right)^{2}=\frac{1}{\lambda}\left(-u_{f}^{3}+3 \lambda u_{f}^{2}+6 u_{f}+6 \lambda \log \left|\frac{\lambda-u_{f}}{\lambda}\right|\right), \\
\eta_{f}=\frac{u_{f}}{\lambda-u_{f}} .
\end{array}\right.
$$

From classical ODE techniques one obtains that for any $\lambda>1$ there exists a unique solution $\left(u_{f}, \eta_{f}\right) \in \mathscr{X}$ such that

$$
\left\{\begin{array}{l}
u_{f}, \eta_{f} \text { are both monotonically decreasing from their crests at } x=0 \text {, and } \\
\qquad \frac{1}{2}\left(3 \lambda-\sqrt{\lambda^{2}+8}\right)<\max _{x \in \mathbb{R}}\left|u_{f}\right|<\lambda .
\end{array}\right.
$$

3.1. Local solutions. Now for any fixed $k>0$ with $k<\lambda$, consider the parameter curve

$$
a=c=k s, \quad b=s, \quad d=\frac{1}{3}-(2 k+1) s .
$$

Thus $b=d$ only when $2(k+1) s=\frac{1}{3}$. So in particular $b \neq d$ when $s$ is small. Moreover we also allow $a, c$ to be negative. 
Similar as before, in this parameter regime we can rewrite (1.3) as

$$
\mathscr{F}(U, s):=\left(\begin{array}{c}
k s u^{\prime \prime}+\lambda s \eta^{\prime \prime}+u-\lambda \eta+\eta u, \\
{\left[\frac{1}{3}-(2 k+1) s\right] \lambda u^{\prime \prime}+k s \eta^{\prime \prime}-\lambda u+\eta+\frac{1}{2} u^{2}}
\end{array}\right)=0,
$$

with $\mathscr{F}: \mathscr{X} \times \mathbb{R} \rightarrow \mathscr{Y}$.

The existence of solitary waves in the parameter regime (3.3) is stated as follows

Theorem 3.1 (Fast waves near the Boussinesq solutions). For any $\lambda>1$, let $k$ be such that $0<k<\lambda$. Suppose that the parameters of (1.3) satisfy (3.3). Then there exist some positive $\delta>0$ and a unique $C^{0}$ solution curve

$$
\mathscr{C}_{\text {loc }}^{\text {fast }}=\left\{\left(u_{s}, \eta_{s}, s\right):|s|<\delta\right\} \subset \mathscr{X} \times \mathbb{R}
$$

to problem (3.4) with the property that

$$
\begin{aligned}
& \left(u_{s}, \eta_{s}\right)=\left(u_{f}, \eta_{f}\right)+O(s) \quad \text { in } \quad \mathscr{X}, \\
& u_{s}, \eta_{s}>0 \quad \text { for } s \geq 0,
\end{aligned}
$$

where $\left(u_{f}, \eta_{f}\right)$ is the unique solution to (3.1) satisfying (3.2).

Proof. Denote $U_{f}:=\left(u_{f}, \eta_{f}\right)$. Working with even functions, direct computation yields that

$$
\mathscr{F}_{U}\left(U_{f}, 0\right)=\left(\begin{array}{cc}
1+\eta_{f} & u_{f}-\lambda \\
\frac{\lambda}{3} \partial_{x}^{2}+u_{f}-\lambda & 1
\end{array}\right): \mathscr{X} \rightarrow \mathscr{Y} .
$$

Suppose that $\mathscr{F}_{U}\left(U_{f}, 0\right)[V]=0$ for some $V=(v, \zeta) \in \mathscr{X}$. Writing out the equations we have

$$
\begin{aligned}
& \left(1+\eta_{f}\right) v+\left(u_{f}-\lambda\right) \zeta=0, \\
& \frac{\lambda}{3} v^{\prime \prime}+\left(u_{f}-\lambda\right) v+\zeta=0 .
\end{aligned}
$$

We can then solve for $\zeta$ in the first equation to obtain a single ODE for $v$

$$
\frac{\lambda}{3} v^{\prime \prime}+\left[\frac{\lambda}{\left(\lambda-u_{f}\right)^{2}}-\left(\lambda-u_{f}\right)\right] v=0 .
$$

Since $\lambda>1$ and $u_{f}$ satisfies (3.2), from classical ODE theory we know that there is only one bounded nontrivial solution to the above equation. On the other hand from the translation invariance of (3.4) we see that $u_{f}^{\prime}$ solves (3.7). From the fact that $u_{f}$ is even, it follows that $\operatorname{ker} \mathscr{F}_{U}\left(U_{f}, 0\right)$ is trivial in $\mathscr{X}$.

The surjectivity of $\mathscr{F}_{U}\left(U_{f}, 0\right)$ can be easily verified since one can effectively solve $\zeta$ in terms of $v$ through an algebraic equation, and then solve an ODE for $v$. Therefore we further conclude that $\mathscr{F}_{U}\left(U_{f}, 0\right)$ is invertible. Thus the existence of the local solution curve and (3.5) follows from the Implicit Function Theorem.

Next let's turn to the sign property. For any $\varepsilon>0$ we can find an $R_{0}>0$ such that

$$
\left|u_{f}(x)\right|,\left|\eta_{f}(x)\right|<\varepsilon \quad \text { for } \quad|x|>R_{0} .
$$


From (3.5) we know that by choosing $s$ sufficiently small,

$$
\begin{cases}u_{s}(x), \eta_{s}(x)>0 & \text { for } \quad|x| \leq R_{0} \\ \left|u_{s}(x)\right|,\left|\eta_{s}(x)\right|<\varepsilon+O(s) & \text { for } \quad|x|>R_{0}\end{cases}
$$

From (3.4) we have

$$
\left(\left[\frac{1}{3}-(2 k+1) s\right] \lambda^{2}-k^{2} s\right) u_{s}^{\prime \prime}-\left[\left(k+\lambda^{2}\right)-\frac{\lambda}{2} u_{s}\right] u_{s}+\left[\lambda+k\left(\lambda-u_{s}\right)\right] \eta_{s}=0 .
$$

So if inf $u_{s}=u_{s}\left(x_{0}\right)<0$, then from (3.8) $\left|x_{0}\right|>R_{0}$. For small $s$, the maximum principle implies that $\eta_{s}\left(x_{0}\right)<0$ and

$$
u_{s}\left(x_{0}\right) \geq \frac{\lambda+k\left(\lambda-u_{s}\left(x_{0}\right)\right)}{k+\lambda^{2}-\lambda u_{s}\left(x_{0}\right) / 2} \eta_{s}\left(x_{0}\right)>\eta_{s}\left(x_{0}\right),
$$

since

$$
0<\frac{\lambda+k\left(\lambda-u_{s}\left(x_{0}\right)\right)}{k+\lambda^{2}-\lambda u_{s}\left(x_{0}\right) / 2}<1
$$

for sufficiently small $\varepsilon$ and $s$.

Since $\eta_{s}\left(x_{0}\right)<0$, we know that inf $\eta_{s}=\eta_{s}\left(x_{1}\right) \leq \eta_{s}\left(x_{0}\right)<0$ for $\left|x_{1}\right|>R_{0}$. Looking at the equation for $\eta_{s}$

$$
\begin{aligned}
\left(\left[\frac{1}{3}-(2 k+1) s\right] \lambda^{2} s-k^{2} s^{2}\right) \eta_{s}^{\prime \prime} & -\left(\left[\frac{1}{3}-(2 k+1) s\right] \lambda^{2}+k s-\left[\frac{1}{3}-(2 k+1) s\right] \lambda u_{s}\right) \eta_{s} \\
+ & \left(\left[\frac{1}{3}-(2 k+1) s\right] \lambda+\lambda k s-\frac{k s}{2} u_{s}\right) u_{s}=0
\end{aligned}
$$

it follows that for $s>0$ small, at $x_{1}$ we have $u_{s}\left(x_{1}\right)<0$, and

$$
\eta_{s}\left(x_{1}\right) \geq \frac{\left[\frac{1}{3}-(2 k+1) s\right] \lambda+\lambda k s-\frac{k s}{2} u_{s}\left(x_{1}\right)}{\left[\frac{1}{3}-(2 k+1) s\right] \lambda^{2}+k s-\left[\frac{1}{3}-(2 k+1) s\right] \lambda u_{s}\left(x_{1}\right)} u_{s}\left(x_{1}\right)>u_{s}\left(x_{1}\right) .
$$

The last estimate holds because for $\varepsilon, s$ sufficiently small the fraction can be made between 0 and 1 . However this would lead to a contradiction since

$$
\inf u_{s}=u_{s}\left(x_{0}\right) \geq \eta_{s}\left(x_{0}\right) \geq \inf \eta_{s}=\eta_{s}\left(x_{1}\right)>u_{s}\left(x_{1}\right) \text {. }
$$

Therefore we have proved that for $s>0$ sufficiently small, $u_{s} \geq 0$. A similar argument yields that $\eta_{s} \geq 0$ as well.

If there is a point $x_{*}$ where $u_{s}\left(x_{*}\right)=0$, then the above argument shows that $\eta_{s}\left(x_{*}\right)=$ 0 , and hence $x_{*}$ is a minimum point for $u_{s}$ and $\eta_{s}$, indicating that $u_{s}^{\prime}\left(x_{*}\right)=\eta_{s}^{\prime}\left(x_{*}\right)=0$. Thus from uniqueness of ODE it must hold that $u_{s}=\eta_{s} \equiv 0$, which is a contradiction. This proves (3.6).

Similar to Section 2, we have the following result establishing the local monotonicity and local uniqueness.

Corollary 3.2 (Local monotonicity and local uniqueness). Denote by $\mathcal{B}_{r}$ the ball of radius $r>0$ in $\left(C^{2}(\mathbb{R}) \cap C_{0}(\mathbb{R})\right) \times\left(C^{2}(\mathbb{R}) \cap C_{0}(\mathbb{R})\right) \times \mathbb{R}$ centered at $\left(u_{f}, \eta_{f}, 0\right)$. There exists $\varepsilon>0$ such that for $s>0$,

$$
\mathcal{F}^{-1}(0) \cap \mathcal{B}_{\varepsilon}=\mathscr{C}_{\text {loc }}^{\text {fast }} \cap \mathcal{B}_{\varepsilon}
$$


In addition, every solution $(u, \eta, s) \in \mathcal{F}^{-1}(0) \cap \mathcal{B}_{\varepsilon}$ is strictly monotone in that for $x>0$,

$$
u^{\prime}<0 \quad \text { and } \quad \eta^{\prime}<0 .
$$

Proof. Similarly as the proof of the sign property in Theorem 3.1. for $(u, \eta, s) \in$ $\mathcal{F}^{-1}(0) \cap \mathcal{B}_{\varepsilon}$, we have $u, \eta>0$. Thus it suffices to check conditions (i) (iii) of Theorem 2.2. Writing (3.9) and (3.10) as

$$
\left\{\begin{array}{l}
\left(\left[\frac{1}{3}-(2 k+1) s\right] \lambda^{2} s-k^{2} s^{2}\right) u^{\prime \prime}+g(u, \eta)=0, \\
\left(\left[\frac{1}{3}-(2 k+1) s\right] \lambda^{2} s-k^{2} s^{2}\right) \eta^{\prime \prime}+f(u, \eta)=0,
\end{array}\right.
$$

direct computation yields that:

$$
\begin{array}{ll}
\frac{\partial g}{\partial \eta}=\lambda+\lambda k-k u, & \frac{\partial f}{\partial u}=\left[\frac{1}{3}-(2 k+1) s\right](\lambda+\eta)+k s(\lambda-u), \\
\frac{\partial g}{\partial u}(0,0)=-\left(\lambda^{2}+k\right), & \frac{\partial f}{\partial \eta}(0,0)=-\left[\frac{1}{3}-(2 k+1) s\right] \lambda^{2}-k s .
\end{array}
$$

When $\varepsilon$ is chosen sufficiently small, conditions (i) (iii) of Theorem 2.2 are satisfied.

Remark 3.1. In the proof above we used a relaxed version of condition (i) which only requires that $\frac{\partial g}{\partial v}\left(u_{\alpha}, v\right), \frac{\partial f}{\partial u}\left(u, v_{\alpha}\right)$ are non-negative for $(u, v) \in[0, \infty) \times[0, \infty)$ where $\left(u_{\alpha}, v_{\alpha}\right)$, where $\left(u_{\alpha}, v_{\alpha}\right)$ are reflection of the solution of the elliptic system with repect to the line $x=\alpha$.

3.2. Nodal pattern and monotone fronts. Now for each fixed $\lambda, k \in \mathbb{R}^{+}$we introduce the set

$$
\mathcal{O}:=\left\{(u, \eta, s) \in \mathscr{X} \times \mathbb{R}^{+}: s \in \Gamma_{1}, u \in \Gamma_{2}\right\}
$$

where

$$
\Gamma_{1}:=\left\{s \in \mathbb{R}^{+}: \frac{\lambda^{2}}{3}-\left[(2 k+1) \lambda^{2}+k^{2}\right] s>0\right\}, \quad \Gamma_{2}:=\left\{u:\|u\|_{C^{0}(\mathbb{R})}<\lambda\right\} .
$$

The intuition for the choice of $\mathcal{O}$ is that $\Gamma_{1}$ is needed for the ellipticity, and $\Gamma_{2}$ provides a sufficient condition to ensure conditions (i) (iii) in Theorem 2.2, in particular the condition (i) for (strict) quasi-monotonicity. Indeed from (3.13) we see that $\partial_{\eta} g, \partial_{u} f>$ 0 when

$$
u<\lambda+\frac{\lambda}{k} \quad \text { and } \quad u<\lambda+\frac{\frac{1}{3}-(2 k+1) s}{k s}(\lambda+\eta)
$$

Moreover, this constraint also allows one to deduce from (3.10) an upper bound for $\eta$

$$
\eta_{s}(0) \leq \frac{\left[\frac{1}{3}-(2 k+1) s\right] \lambda+\left(\lambda-\frac{u_{s}(0)}{2}\right) k s}{\left[\frac{1}{3}-(2 k+1) s\right] \lambda\left(\lambda-u_{s}(0)\right)+k s} u_{s}(0) .
$$

Constraint $\Gamma_{2}$ can also be understood as a "no stagnation" condition and indicates that the particles travel behind the wave. 
From Theorem 3.1 and Corollary 3.2 we are led to consider the following nodal property:

$$
\begin{array}{ccc}
u>0, & \eta>0 & \text { in } \mathbb{R}, \\
u^{\prime}<0, & \eta^{\prime}<0 & \text { in } \mathbb{R}^{+},
\end{array}
$$

Similarly to the previous section, we can prove that the above nodal property persists on the solution curve. The proof follows along the same line as the one in Lemma 2.6. and hence we omit it.

Lemma 3.1 (Nodal property). If $\mathcal{K}$ is any connected subset of $\mathcal{O} \cap \mathscr{F}^{-1}(0)$ that contains $\mathscr{C}_{\text {loc }}^{\text {fast }}$, then every $(u, \eta, \lambda) \in \mathcal{K}$ exhibits (3.16).

The next step regards the nonexistence of monotone fronts, which will provide useful information for the global theory. As in Section 2.4, we define the concept of monotone fronts as follows.

Definition 3.3. Let $s \in \Gamma_{1}, u \in \Gamma_{2}$. we say $(u, \eta, \lambda)$ is a monotone front solution of (3.4) if $(u, \eta) \in C_{\mathrm{b}}^{2}(\mathbb{R}) \times C_{\mathrm{b}}^{2}(\mathbb{R})$, and

$$
\lim _{x \rightarrow+\infty}(u(x), \eta(x))=(0,0), \quad \text { and } \quad u>0, \quad \eta>0, \quad u^{\prime} \leq 0, \quad \eta^{\prime} \leq 0 \quad \text { in } \mathbb{R} .
$$

Lemma 3.2 (Nonexistence of monotone fronts). If $s \in \Gamma_{1}, u \in \Gamma_{2}$ then system (3.4) does not admit any monotone front solution in the sense of (3.17).

Proof. The proof is similar to Lemma 2.7 but the algebra is simpler. Suppose $(u, \eta)$ is a monotone front solution to (3.4). Let

$$
(\bar{u}, \bar{\eta}):=\lim _{x \rightarrow-\infty}(u(x), \eta(x)) .
$$

So $\bar{u}, \bar{\eta}>0$. Evaluating (3.4) at $x \rightarrow-\infty$ implies that

$$
\bar{u}=\frac{3 \lambda-\sqrt{\lambda^{2}+8}}{2}
$$

and

$$
\bar{\eta}=\frac{\bar{u}}{\lambda-\bar{u}} .
$$

Multiplying the first equation in (3.4) by $\eta^{\prime}$, the second equation by $u^{\prime}$, and then summing them gives

$$
\begin{aligned}
& {\left[\frac{\left(\frac{1}{3}-(2 k+1) s\right) \lambda}{2}\left(u^{\prime}\right)^{2}+\frac{\lambda s}{2}\left(\eta^{\prime}\right)^{2}+k s u^{\prime} \eta^{\prime}+u \eta-\frac{\lambda}{2} u^{2}-\frac{\lambda}{2} \eta^{2}+\frac{1}{6} u^{3}\right]^{\prime}} \\
& +u \eta \eta^{\prime}=0
\end{aligned}
$$

Write $u \eta \eta^{\prime}=\left(\frac{1}{2} u \eta^{2}\right)^{\prime}-\frac{1}{2} u^{\prime} \eta^{2}$, by definition of monotone front, we have $u^{\prime} \eta^{2} \leq 0$ and thus

$$
\bar{u} \bar{\eta}-\frac{\lambda}{2} \bar{u}^{2}-\frac{\lambda}{2} \bar{\eta}^{2}+\frac{1}{6} \bar{u}^{3}+\frac{1}{2} \bar{u} \bar{\eta}^{2} \geq 0
$$

From (3.19), this can be reduced to

$$
(\lambda-\bar{u})\left(\frac{\bar{u}}{3}-\lambda\right)+1 \geq 0
$$


and from (3.18) we finally have

$$
\frac{1}{12}\left(-\lambda+\sqrt{\lambda^{2}+8}\right)\left(-3 \lambda-\sqrt{\lambda^{2}+8}\right)+1 \geq 0
$$

the above inequality holds only when $\lambda \leq 1$, which contradicts the fact that $\lambda>1$.

3.3. Global continuation. As in Section 2.5, with Lemma 3.1] and 3.2, we obtain the following global solution curve:

Theorem 3.4. There exists a curve $\mathscr{C}^{\text {fast }}$ containing $\mathscr{C}_{\text {loc }}^{\text {fast}}$, which admits a global $C^{0}$ parametrization

$$
\mathscr{C}^{\text {fast }}:=\{(u(t), \eta(t), s(t)): t \in(0, \infty)\} \subset \mathcal{O} \cap \mathscr{F}^{-1}(0)
$$

with $\lim _{t \searrow 0}(u(t), \eta(t), s(t))=\left(u_{f}, \eta_{f}, 0\right)$ and satisfies the following property:

(a) (Symmetry and monotonicity) Each solution on $\mathscr{C}^{\text {fast }}$ is even and

$$
\begin{aligned}
& u(t)>0 \quad \eta(t)>0 \quad \text { on } \mathbb{R}, \\
& \partial_{x} u(t)<0 \quad \partial_{x} \eta(t)<0 \quad \text { on } \mathbb{R}^{+} .
\end{aligned}
$$

(b) (Loss of ellipticity or stagnation limit) Following $\mathscr{C}^{\text {fast }}$ to its extreme, either the system loses ellipticity in that

$$
\lim _{t \rightarrow \infty} s(t)=\frac{\lambda^{2}}{3\left((2 k+1) \lambda^{2}+k^{2}\right)},
$$

or we encounter waves that are arbitrarily close to having a stagnation point

$$
\lim _{t \rightarrow \infty} \inf _{x \in \mathbb{R}}(\lambda-u(t))=0 .
$$

Proof. We will only focus on proving (b). Since $\lim _{t \rightarrow \infty} s(t)>0$, so if (3.22) is false, we can find a sequence $\left\{t_{n}\right\} \rightarrow \infty$ with the corresponding solutions denoted by $\left(u_{n}, \eta_{n}, s_{n}\right) \in \mathcal{O} \cap \mathscr{F}^{-1}(0)$ such that as $n \rightarrow \infty$,

$$
s_{n} \rightarrow s_{*}<\frac{\lambda^{2}}{3\left((2 k+1) \lambda^{2}+k^{2}\right)}, \quad \text { either }\left\|\left(u_{n}, \eta_{n}\right)\right\|_{\mathscr{X}} \rightarrow \infty \text { or }\left\|u_{n}\right\|_{C^{0}} \rightarrow \lambda .
$$

Recall from (3.15) the upper bound for $\eta_{n}$

$$
\bar{\eta}_{n} \leq \frac{\bar{s}_{n} \lambda+\left(\lambda-\frac{\bar{u}_{n}}{2}\right) k s_{n}}{\bar{s}_{n} \lambda\left(\lambda-\bar{u}_{n}\right)+k s_{n}} \bar{u}_{n} \leq \frac{2 \bar{s}_{n} \lambda+\lambda k s_{n}}{2 k s_{n}} \lambda,
$$

where $\bar{s}_{n}:=\frac{1}{3}-(2 k+1) s_{n}$. Elliptic regularity then asserts that the latter alternative can be replaced by

proving (3.23).

$$
\left\|u_{n}\right\|_{C^{0}} \rightarrow \lambda
$$

\section{ACKNowledGements}

R. M. Chen and J. Jin are supported in part by the NSF grants DMS-1613375 and DMS-1907584. 


\section{Appendix A. Proofs from Section 2.1}

In this appendix we provide the proofs of the properties for the stationary wave problem (2.1) stated in Section 2.1.

Proof of Lemma 2.1. If there is an $x_{1}$ such that $\eta\left(x_{1}\right) \geq 0$. This property, and the fact that $\eta(x) \rightarrow 0$ as $x \rightarrow \pm \infty$, imply that $\eta$ has a non-negative maximum on $\mathbb{R}$. Suppose that this maximum is at $x_{0}$. Then from (2.1), either $\eta\left(x_{0}\right)=u\left(x_{0}\right)=0$ or $\eta^{\prime \prime}\left(x_{0}\right)>0$. The latter being impossible at a maximum, we conclude the former two equalities. Also, $\eta^{\prime}\left(x_{0}\right)=0$, and from (2.2),$u^{\prime}\left(x_{0}\right)=0$. But then, since $\eta, \eta^{\prime}, u$, and $u^{\prime}$ all vanish at $x_{0}$, the uniqueness theorem for ODE's implies that $\eta(x)=u(x)=0$ for all $x$, contradicting the definition of a solitary wave, which must be non-constant. This completes the proof of Lemma 2.1.

A quick application of the maximum principle also yields

Lemma A.1. Let $(u, \eta)$ be a solitary wave solution. Then

(a) $(-u, \eta)$ is also a solitary wave solution.

(b) If $u \geq 0$ then $u(x)>0$.

(c) If $u \leq 0$ then $u(x)<0$.

Proof. Part (a) follows directly from (2.1). For (b), suppose that $u(x) \geq 0$ and that $u\left(x_{1}\right)=0$ for some $x_{1}$. Since $u$ is non-negative it must be the case that $u^{\prime}\left(x_{1}\right)=0$. Thus, by uniqueness of the constant solution $\left(u, u^{\prime}\right)=(0,0)$ of

$$
-a u^{\prime \prime}=u(1+\eta)
$$

we conclude that $u(x) \equiv 0$. In this case, $\eta^{\prime \prime}=\eta$ on $\mathbb{R}$. But since the orbit is homoclinic, $\eta$ must be bounded, which implies that $\eta(x)=0$ for all $x$ as well, and this contradicts the definition of solitary waves and Lemma 2.1. Part (c) follows by a similar argument.

Proof of Proposition [2.1. The proof part (i) makes use of function

$$
h=u-\sqrt{2} \eta,
$$

which satisfies

$$
\beta^{2} h^{\prime \prime}=\left(1-\frac{u}{\sqrt{2}}\right) h .
$$

If $(u, \eta)$ is a solitary wave solution with $u<\sqrt{2}$, then $1-u / \sqrt{2}>0$. Then from maximum principle we know that $h \equiv 0$.

Substituting $h=u-\sqrt{2} \eta=0$ into (2.2) we get

$$
\beta^{2}\left(\eta^{\prime}\right)^{2}=\eta^{2}+\frac{2}{3} \eta^{3}
$$

which is the classical steady $\mathrm{KdV}$ equation. The solution is given by

$$
\eta_{0}(x)=-\frac{3}{2} \operatorname{sech}^{2}\left(\frac{x}{2 \beta}\right), \quad \text { and hence } \quad u_{0}^{-}(x)=-\frac{3 \sqrt{2}}{2} \operatorname{sech}^{2}\left(\frac{x}{2 \beta}\right) .
$$

The proof of (ii) makes use of the functional $w=u+\sqrt{2} \eta$, which satisfies

$$
\beta^{2} w^{\prime \prime}=\left(1+\frac{u}{\sqrt{2}}\right) w .
$$


The remainder of the proof follows the same argument as before, with $w$ replacing $h$.

\section{REFERENCES}

[1] Ablowitz, M. J., Haut, T. S., Asymptotic expansions for solitary gravity-capillary waves in two and three dimensions, Proc. R. Soc. Lond. Ser. A Math. Phys. Eng. Sci. 465 (2009), 2725-2749.

[2] Adelaide, A., Walsh, S., Solitary water waves with discontinuous vorticity, J. Math. Pures Appl., 124 (2019), 220-272.

[3] Amick, C. J., Regularity and uniqueness of solutions to the Boussinesq system of equations, J. Differential Equaitons, 54 (1984), 231-247.

[4] Amick, C. J., Toland, J. F., On periodic water-waves and their convergence to solitary waves in the long-wave limit, Philos. Trans. Roy. Soc. London Ser. A, 303 (1981), 633-669.

[5] Amick, C. J., Toland, J. F., On sollitary water-waves of finite amplitude, Arch. Ration. Mech. Anal., 76 (1981), 9-95.

[6] Bao, E.S., Chen, R.M., Liu, Q., Existence and symmetry of ground states to a Boussinesq abcd system, Arch. Ration. Mech. Anal., 216 (2015) 569-591.

[7] Benjamin, T. B. , Bona, J. L., Bose, D. K., Solitary-wave solutions of nonlinear problems, Philos. Trans. Roy. Soc. London Ser. A, 331 (1990), 195-244.

[8] Benjamin, T. B. , Bona, J. L., Mahoney,J. J., Model equations for long waves in nonlinear dispersive systems, Phil. Trans. Roy. Soc. London Ser. A, 227 (1972), 47-78.

[9] Bona, J.L., Chen, M., Saut, J.C., Boussinesq equations and other systems for small-amplitude long waves in nonlinear dispersive media. I. Derivation and linear theory, J. Nonlinear Sci., 12 (2002), 283-318.

[10] Bona, J.L., Colin, T., Lannes, D., Long wave approximations for water waves, Arch. Ration. Mech. Anal., 178 (2005), 373-410.

[11] Boussinesq, J.,, Théorie de l'intumescence liquide appelée onde solitaire ou de translation se propageant dans un canal rectangulaire, Comptes Rendus Acad. Sci. Paris, 72 (1871), 755-759.

[12] Boussinesq, J., Théorie générale des mouvements qui sont propagés dans un canal rectangulaire horizontal, Comptes Rendus Acad. Sci. Paris, 73 (1871), 256-260.

[13] Busca, J., Sirakov, B., Symmetry results for semilinear elliptic systems in the whole space, $J$. Differential Equations, 163 (2000), 41-56.

[14] Chazel, F., Influence of topography on water waves, ESAIM: M2AN, 41 (2007), 771-799.

[15] Chen, M., Solitary-wave and multi-pulsed traveling-wave solutions of Boussinesq systems, Appl. Anal., 75 (2000), 213-240.

[16] Chen, M., Nguyen, N., Sun, S.-M., Solitary-wave solutions to Boussinesq systems with large surface tension, Discrete Contin. Dyn. Syst. Ser. S., 26 (2009), 1153-1184.

[17] Chen, M., Nguren, N., Sun, S.-M., Existence of Traveling-wave Solutions to Boussinesq Systems, Diff. $\mathscr{G}$ Integral Eq., 24 (2011), 895-908.

[18] Chen, R. M., Hasting, S., Troy, W. C., Solitary waves of the Boussinesq abcd system, preprint.

[19] Chen, R. M., Walsh, S., Wheeler, M., Existence and qualitative theory for stratified solitary water waves, Ann. Inst. H. Poincaré Anal. Non Linéaire, 35 (2018), 517-576.

[20] Chen, R. M., Walsh, S., Wheeler, M., Global bifurcation for monotone fronts of elliptic equations, arXiv preprint arXiv:2005.00651 (2020).

[21] Chen, R. M., Walsh, S., Wheeler, M., Global bifurcation of anti-plane shear fronts, J. Nonlinear Sci., 31 (2021), no. 2, 28.

[22] Craig, W., An existence theory for water waves, and Boussinesq and Korteweg-de Vries scaling limits, Commun. PDEs, 10 (1985), 787-1003.

[23] Daripa, P., Dash R. K. , A class of model equations for bi-directional propagation of capillarygravity waves, Internat. J. Engrg. Sci., 41 (2003), 201-218.

[24] Friedrichs, K.O. And Hyers, D.H., The existence of solitary waves, Comm. Pure Appl. Math., 7 (1954), 517-550.

[25] Hogancamp, T., Broadening global families of anti-plane shear equilibria, arXiv preprint arXiv:2101.07909 (2020). 
[26] Kano, K., Nishida, T., A mathematical justification for Korteweg-de Vries equation and Boussinesq equation of water surface waves, Osaka J. Math., 23 (1986), 389-413.

[27] Kirchgassner, K., Wave solutions of reversible systems and applications, J. Diff. Eq., 45 (1982), $113-127$.

[28] Korteweg, D. J., DE VRIEs,G., On the change of form of long waves advancing in a rectangular canal and on a new type of long stationary waves, Phil. Mag., 39 (1895), 422-443.

[29] Lavrentiev, M.A., I. On the theory of long waves. II. A contribution to the theory of long waves, Amer. Math. Soc. Transl., (1954), 53.

[30] Peregrine, D. H., Equations for water waves and the approximation behind them, in Waves on beaches and resulting sediment transport; proceedings of an advanced seminar conducted by the Mathematics Research Center, New York, Academic Press, (1972), 95-121.

[31] Rayleigh, L., On Waves, Phil. Mag., 1 (1876), 257-279.

[32] Russell, J. S., Report on waves, in 14th meeting of British Association for Advancement of Science, vol. 311, 1844, p. 390.

[33] Sachs, R., On the existence of small amplitude waves with strong surface tension, J. Differential. Equ., 90 (1991), 31-51.

[34] Saut, J, -C., Xu, L., The Cauchy problem on large time for surface waves Boussinesq systems, J. Math. Pures Appl., 97 (2012), 635-662.

[35] Schonbek, M. E., Existence of solutions for the Boussinesq system of equations, J. Differential Equations, 42 (1981), 325-352.

[36] Sinambela, D., Large-amplitude solitary waves in two-layer density stratified water, arXiv preprint arXiv:2012.00142 (2020).

[37] Ter-Krikorov, A. M., The existence of periodic waves which degenerate into a solitary wave, $J$. Appl. Math. Mech., 24 (1960), 930-949.

[38] Toland, J. F., Solitary wave solutions for a model of the two-way propagation of water waves in a channel, Math. Proc. Camb. Phil. Soc. 90 (1981), 343-360.

[39] Ursell, F., The long-wave paradox in the theory of gravity waves, Proc. Cambridge Phil. Soc., 49 (1953), 685-694.

[40] WheELER, M. Solitary water waves of large amplitude generated by surface pressure, Arch. Ration. Mech. Anal., 218 (2015) 1131-1187.

Department of Mathematics, University of Pittsburgh, Pittsburgh PA 15260

Email address: mingchen@pitt.edu

Department of Mathematics, University of Pittsburgh, Pittsburgh PA 15260

Email address: jij50@pitt.edu 